\title{
A Sturm-Liouville Problem with a Discontinuous Coefficient and Containing an Eigenparameter in the Boundary Condition
}

\author{
Erdoğan Şen ${ }^{1,2}$ \\ ${ }^{1}$ Department of Mathematics, Faculty of Arts and Science, Namik Kemal University, 59030 Tekirdağ, Turkey \\ ${ }^{2}$ Department of Mathematics Engineering, Istanbul Technical University, Maslak, 34469 Istanbul, Turkey
}

Correspondence should be addressed to Erdoğan Şen; erdogan.math@gmail.com

Received 10 April 2013; Accepted 18 July 2013

Academic Editor: Ashok Chatterjee

Copyright @ 2013 Erdoğan Şen. This is an open access article distributed under the Creative Commons Attribution License, which permits unrestricted use, distribution, and reproduction in any medium, provided the original work is properly cited.

\begin{abstract}
We study a Sturm-Liouville operator with eigenparameter-dependent boundary conditions and transmission conditions at two interior points. We give an operator-theoretic formulation, construct fundamental solutions, investigate some properties of the eigenvalues and corresponding eigenfunctions of the discontinuous Sturm-Liouville problem and then obtain asymptotic formulas for the eigenvalues and eigenfunctions and find Green function of the discontinuous Sturm-Liouville problem.
\end{abstract}

\section{Introduction}

In recent years, more and more researchers are interested in the discontinuous Sturm-Liouville problem for its application in physics (see [1-16]). Such problems are connected with discontinuous material properties, such as heat and mass transfer, varied assortment of physical transfer problems, vibrating string problems when the string loaded additionally with point masses, and diffraction problems. Moreover, there has been a growing interest in Sturm-Liouville problems with eigenparameter-dependent boundary conditions; that is, the eigenparameter appears not only in the differential equations but also in the boundary conditions of the problems (see [1$16]$ and corresponding bibliography).

In this paper, following [8] we consider the boundary value problem for the differential equation

$$
\tau u:=-u^{\prime \prime}+q(x) u=\lambda \omega(x) u,
$$

for $x \in\left[-1, h_{1}\right) \cup\left(h_{1}, h_{2}\right) \cup\left(h_{2}, 1\right]$ (i.e., $x$ belongs to $[-1,1]$, but the two inner points are $x=h_{1}$ and $x=h_{2}$ ), where $q(x)$ is a real-valued function, continuous in $\left[-1, h_{1}\right),\left(h_{1}, h_{2}\right)$, and $\left(h_{2}, 1\right]$ with the finite limits $q\left( \pm h_{1}\right)=\lim _{x \rightarrow \pm h_{1}}, q\left( \pm h_{2}\right)=$ $\lim _{x \rightarrow \pm h_{2}} ; \omega(x)$ is a discontinuous weight function such that $\omega(x)=\omega_{1}^{2}$ for $x \in\left[-1, h_{1}\right), \omega(x)=\omega_{2}^{2}$ for $x \in\left(h_{1}, h_{2}\right)$, and $\omega(x)=\omega_{3}^{2}$ for $x \in\left(h_{2}, 1\right], \omega>0$ together with the standard boundary condition at $x=-1$

$$
L_{1} u:=u^{\prime}(-1)=0,
$$

the spectral parameter-dependent boundary condition at $x=1$

$$
L_{2} u:=\lambda\left(\beta_{1}^{\prime} u(1)-\beta_{2}^{\prime} u^{\prime}(1)\right)+\left(\beta_{1} u(1)-\beta_{2} u^{\prime}(1)\right)=0,
$$

and the four transmission conditions at the points of discontinuity $x=h_{1}$ and $x=h_{2}$

$$
\begin{gathered}
L_{3} u:=\gamma_{1} u\left(h_{1}-0\right)-\delta_{1} u\left(h_{1}+0\right)=0, \\
L_{4} u:=\gamma_{2} u^{\prime}\left(h_{1}-0\right)-\delta_{2} u^{\prime}\left(h_{1}+0\right)=0, \\
L_{5} u:=\gamma_{3} u\left(h_{2}-0\right)-\delta_{3} u\left(h_{2}+0\right)=0, \\
L_{6} u:=\gamma_{4} u^{\prime}\left(h_{2}-0\right)-\delta_{4} u^{\prime}\left(h_{2}+0\right)=0,
\end{gathered}
$$

in the Hilbert space $L_{2}\left(-1, h_{1}\right) \oplus L_{2}\left(h_{1}, h_{2}\right) \oplus L_{2}\left(h_{2}, 1\right)$, where $\lambda \in \mathbb{C}$ is a complex spectral parameter; and all coefficients of the boundary and transmission conditions are real constants. We assume that $\beta_{2}^{\prime} \neq 0$ and $\left|\beta_{1}\right|+\left|\beta_{2}\right| \neq 0$. Moreover, we will assume that $\rho:=\beta_{1}^{\prime} \beta_{2}-\beta_{1} \beta_{2}^{\prime}>0$. Some special cases of this problem arise after application of the method of separation 
of variables to the diverse assortment of physical problems, heat and mass transfer problems (e.g., see [11]), vibrating string problems when the string was loaded additionally with point masses (e.g., see [11]), and a thermal conduction problem for a thin laminated plate.

\section{Operator-Theoretic Formulation of the Problem}

In this section, we introduce a special inner product in the Hilbert space $\left(L_{2}\left(-1, h_{1}\right) \oplus L_{2}\left(h_{1}, h_{2}\right) \oplus L_{2}\left(h_{2}, 1\right)\right) \oplus \mathbb{C}$ and define a linear operator $A$ in it so that the problem (1)-(5) can be interpreted as the eigenvalue problem for $A$. To this end, we define a new Hilbert space inner product on $H$ := $\left(L_{2}\left(-1, h_{1}\right) \oplus L_{2}\left(h_{1}, h_{2}\right) \oplus L_{2}\left(h_{2}, 1\right)\right) \oplus \mathbb{C}$ by

$$
\begin{aligned}
\langle F, G\rangle_{H}= & \omega_{1}^{2} \int_{-1}^{h_{1}} f(x) \overline{g(x)} d x+\omega_{2}^{2} \frac{\delta_{1} \delta_{2}}{\gamma_{1} \gamma_{2}} \int_{h_{1}}^{h_{2}} f(x) \overline{g(x)} d x \\
& +\omega_{3}^{2} \frac{\delta_{1} \delta_{2} \delta_{3} \delta_{4}}{\gamma_{1} \gamma_{2} \gamma_{3} \gamma_{4}} \int_{h_{2}}^{1} f(x) \overline{g(x)} d x \\
& +\frac{\delta_{1} \delta_{2} \delta_{3} \delta_{4}}{\rho \gamma_{1} \gamma_{2} \gamma_{3} \gamma_{4}} f_{1} \overline{g_{1}}
\end{aligned}
$$

for $F=\left(\begin{array}{c}f(x) \\ f_{1}\end{array}\right)$ and $G=\left(\begin{array}{c}g(x) \\ g_{1}\end{array}\right) \in H$. For convenience, we will use the notations

$$
\begin{aligned}
& R_{1}(u):=\beta_{1} u(1)-\beta_{2} u^{\prime}(1), \\
& R_{1}^{\prime}(u):=\beta_{1}^{\prime} u(1)-\beta_{2}^{\prime} u^{\prime}(1) .
\end{aligned}
$$

In this Hilbert space, we construct the operator $A: H \rightarrow H$ with domain

$$
\begin{aligned}
& D(A)=\left\{F=\left(\begin{array}{c}
f(x) \\
f_{1}
\end{array}\right) \mid f(x), f^{\prime}(x)\right. \\
& \text { are absolutely continuous in }\left[1, h_{1}\right] \cup\left[h_{1}, h_{2}\right] \\
& \cup\left[h_{2}, 1\right] \text { and have finite limits } \\
& f\left(h_{1} \pm 0\right), f\left(h_{2} \pm 0\right), f^{\prime}\left(h_{1} \pm 0\right), f^{\prime}\left(h_{2} \pm 0\right) \text {, } \\
& \tau f \in L_{2}\left(-1, h_{1}\right) \oplus L_{2}\left(h_{1}, h_{2}\right) \oplus L_{2}\left(h_{2}, 1\right), \\
& L_{1} f=L_{3} f=L_{4} f=L_{5} f=L_{6} f=0 \text {, } \\
& \left.f_{1}=R_{1}^{\prime}(f)\right\}
\end{aligned}
$$

which acts by the rule

$$
\begin{aligned}
& A F=\left(\begin{array}{c}
\frac{1}{\omega(x)}\left[-f^{\prime \prime}+q(x) f\right] \\
-R_{1}(f)
\end{array}\right) \\
& \text { with } F=\left(\begin{array}{c}
f(x) \\
R_{1}^{\prime}(f)
\end{array}\right) \in D(A) \text {. }
\end{aligned}
$$

Thus we can pose the boundary value transmission problem (1)-(7) in $H$ as

$$
A U=\lambda U, \quad U:=\left(\begin{array}{c}
u(x) \\
R_{1}^{\prime}(u)
\end{array}\right) \in D(A) .
$$

It is readily verified that the eigenvalues of $A$ coincide with those of the problem (1)-(7).

Theorem 1. The operator $A$ is symmetric.

Proof. Let $F=\left(\begin{array}{c}f(x) \\ R_{1}^{\prime}(f)\end{array}\right)$ and $G=\left(\begin{array}{c}g(x) \\ R_{1}^{\prime}(g)\end{array}\right)$ be arbitrary elements of $D(A)$. Twice integrating by parts, we find

$$
\begin{aligned}
\langle A F, G\rangle_{H}-\langle F, A G\rangle_{H} \\
=W\left(f, \bar{g} ; h_{1}-0\right)-W(f, \bar{g} ;-1) \\
+\frac{\delta_{1} \delta_{2}}{\gamma_{1} \gamma_{2}}\left(W\left(f, \bar{g} ; h_{2}-0\right)-W\left(f, \bar{g} ; h_{1}+0\right)\right) \\
+\frac{\delta_{1} \delta_{2} \delta_{3} \delta_{4}}{\gamma_{1} \gamma_{2} \gamma_{3} \gamma_{4}}\left(W(f, \bar{g} ; 1)-W\left(f, \bar{g} ; h_{2}+0\right)\right) \\
+\frac{\delta_{1} \delta_{2} \delta_{3} \delta_{4}}{\rho \gamma_{1} \gamma_{2} \gamma_{3} \gamma_{4}}\left(R_{1}^{\prime}(f) R_{1}(\bar{g})-R_{1}(f) R_{1}^{\prime}(\bar{g})\right),
\end{aligned}
$$

where, as usual, $W(f, g ; x)$ denotes the Wronskian of $f$ and $g$; that is,

$$
W(f, g ; x):=f(x) g^{\prime}(x)-f^{\prime}(x) g(x) .
$$

Since $F, G \in D(A)$, the first components of these elements, that is, $f$ and $g$, satisfy the boundary condition (2). From this fact, we easily see that

$$
W(f, \bar{g} ;-1)=0 .
$$

Further, as $f$ and $g$ also satisfy both transmission conditions, we obtain

$$
\begin{gathered}
W\left(f, \bar{g} ; h_{1}-0\right)=\frac{\delta_{1} \delta_{2}}{\gamma_{1} \gamma_{2}} W\left(f, \bar{g} ; h_{1}+0\right), \\
W\left(f, \bar{g} ; h_{2}-0\right)=\frac{\delta_{1} \delta_{2} \delta_{3} \delta_{4}}{\gamma_{1} \gamma_{2} \gamma_{3} \gamma_{4}} W\left(f, \bar{g} ; h_{2}+0\right) .
\end{gathered}
$$

Moreover, the direct calculations give

$$
R_{1}^{\prime}(f) R_{1}(\bar{g})-R_{1}(f) R_{1}^{\prime}(\bar{g})=-\rho W(f, \bar{g} ; 1) .
$$

Now, inserting (15)-(17) in (13), we have

$$
\langle A F, G\rangle_{H}=\langle F, A G\rangle_{H} \quad(F, G \in D(A)),
$$

and so $A$ is symmetric.

Recalling that the eigenvalues of (1)-(7) coincide with the eigenvalues of $A$, we have the next corollary.

Corollary 2. All eigenvalues of (1)-(7) are real.

Since all eigenvalues are real, it is enough to study only the real-valued eigenfunctions. Therefore, we can now assume that all eigenfunctions of (1)-(7) are real-valued. 


\section{Asymptotic Formulas for Eigenvalues and Fundamental Solutions}

Let us define fundamental solutions

$$
\begin{gathered}
\phi(x, \lambda)= \begin{cases}\phi_{1}(x, \lambda), & x \in\left[-1, h_{1}\right), \\
\phi_{2}(x, \lambda), & x \in\left(h_{1}, h_{2}\right), \\
\phi_{3}(x, \lambda), & x \in\left(h_{2}, 1\right],\end{cases} \\
\chi(x, \lambda)= \begin{cases}\chi_{1}(x, \lambda), & x \in\left[-1, h_{1}\right), \\
\chi_{2}(x, \lambda), & x \in\left(h_{1}, h_{2}\right), \\
\chi_{3}(x, \lambda), & x \in\left(h_{2}, 1\right],\end{cases}
\end{gathered}
$$

of (1) by the following procedure. We first consider the next initial value problem:

$$
\begin{gathered}
-u^{\prime \prime}+q(x) u=\lambda \omega_{1}^{2} u, \quad x \in\left[-1, h_{1}\right], \\
u(-1)=1, \\
u^{\prime}(-1)=0 .
\end{gathered}
$$

By virtue of [1, Theorem 1.5], the problem (20)-(22) has a unique solution $u=\phi_{1}(x, \lambda)$ which is an entire function of $\lambda \in \mathbb{C}$ for each fixed $x \in\left[-1, h_{1}\right]$. Similarly,

$$
\begin{aligned}
-u^{\prime \prime}+q(x) u & =\lambda \omega_{2}^{2} u, \quad x \in\left[h_{1}, h_{2}\right], \\
u\left(h_{1}\right) & =\frac{\gamma_{1}}{\delta_{1}} \phi_{1}\left(h_{1}, \lambda\right), \\
u^{\prime}\left(h_{1}\right) & =\frac{\gamma_{2}}{\delta_{2}} \phi_{1}^{\prime}\left(h_{1}, \lambda\right),
\end{aligned}
$$

have a unique solution $u=\phi_{2}(x, \lambda)$ which is an entire function of $\lambda \in \mathbb{C}$ for each fixed $x \in\left[h_{1}, h_{2}\right]$. Continuing in this manner,

$$
\begin{aligned}
-u^{\prime \prime}+q(x) u & =\lambda \omega_{3}^{2} u, \quad x \in\left[h_{2}, 1\right], \\
u\left(h_{2}\right) & =\frac{\gamma_{3}}{\delta_{3}} \phi_{2}\left(h_{2}, \lambda\right), \\
u^{\prime}\left(h_{2}\right) & =\frac{\gamma_{4}}{\delta_{4}} \phi_{2}^{\prime}\left(h_{2}, \lambda\right),
\end{aligned}
$$

have a unique solution $u=\phi_{3}(x, \lambda)$ which is an entire function of $\lambda \in \mathbb{C}$ for each fixed $x \in\left[h_{2}, 1\right]$. Slightly modifying the method of [1, Theorem 1.5$]$, we can prove the initial value problem

$$
\begin{gathered}
-u^{\prime \prime}+q(x) u=\lambda \omega_{3}^{2} u, \quad x \in\left[h_{2}, 1\right], \\
u(1)=\beta_{2}^{\prime} \lambda+\beta_{2}, \\
u^{\prime}(1)=\beta_{1}^{\prime} \lambda+\beta_{1},
\end{gathered}
$$

have a unique solution $u=\chi_{3}(x, \lambda)$ which is an entire function of spectral parameter $\lambda \in \mathbb{C}$ for each fixed $x \in$ $\left[h_{2}, 1\right]$. Similarly,

$$
\begin{aligned}
-u^{\prime \prime}+q(x) u & =\lambda \omega_{2}^{2} u, \quad x \in\left[h_{1}, h_{2}\right], \\
u\left(h_{2}\right) & =\frac{\delta_{3}}{\gamma_{3}} \chi_{3}\left(h_{2}, \lambda\right), \\
u^{\prime}\left(h_{2}\right) & =\frac{\delta_{4}}{\gamma_{4}} \chi_{3}^{\prime}\left(h_{2}, \lambda\right),
\end{aligned}
$$

have a unique solution $u=\chi_{2}(x, \lambda)$ which is an entire function of $\lambda \in \mathbb{C}$ for each fixed $x \in\left[h_{1}, h_{2}\right]$. Continuing in this manner,

$$
\begin{gathered}
-u^{\prime \prime}+q(x) u=\lambda \omega_{3}^{2} u, \quad x \in\left[-1, h_{1}\right], \\
u\left(h_{1}\right)=\frac{\delta_{1}}{\gamma_{1}} \chi_{2}\left(h_{1}, \lambda\right) \\
u^{\prime}\left(h_{1}\right)=\frac{\delta_{2}}{\gamma_{2}} \chi_{2}^{\prime}\left(h_{1}, \lambda\right)
\end{gathered}
$$

have a unique solution $u=\chi_{1}(x, \lambda)$ which is an entire function of $\lambda \in \mathbb{C}$ for each fixed $x \in\left[-1, h_{1}\right]$.

By virtue of (21) and (22), the solution $\phi(x, \lambda)$ satisfies the first boundary condition (2). Moreover, by (24), (25), (27), and $(28), \phi(x, \lambda)$ satisfies also transmission conditions (4)(7). Similarly, by (30), (31), (33), (34), (36), and (37) the other solution $\chi(x, \lambda)$ satisfies the second boundary condition (3) and transmission conditions (4)-(7). It is well known from the theory of ordinary differential equations that each of the Wronskians $\Delta_{1}(\lambda)=W\left(\phi_{1}(x, \lambda), \chi_{1}(x, \lambda)\right), \Delta_{2}(\lambda)=$ $W\left(\phi_{2}(x, \lambda), \chi_{2}(x, \lambda)\right)$, and $\Delta_{3}(\lambda)=W\left(\phi_{3}(x, \lambda), \chi_{3}(x, \lambda)\right)$ is independent of $x$ in $\left[-1, h_{1}\right],\left[h_{1}, h_{2}\right]$, and $\left[h_{2}, 1\right]$, respectively.

Lemma 3. The equality $\Delta_{1}(\lambda)=\left(\delta_{1} \delta_{2} / \gamma_{1} \gamma_{2}\right) \Delta_{2}(\lambda)=$ $\left(\delta_{1} \delta_{2} \delta_{3} \delta_{4} / \gamma_{1} \gamma_{2} \gamma_{3} \gamma_{4}\right) \Delta_{3}(\lambda)$ holds for each $\lambda \in \mathbb{C}$.

Proof. Since the above Wronskians are independent of $x$, using (27), (28), (30), (31), (33), (34), (36), and (37), we find

$$
\begin{aligned}
\Delta_{1}(\lambda)= & \phi_{1}\left(h_{1}, \lambda\right) \chi_{1}^{\prime}\left(h_{1}, \lambda\right)-\phi_{1}^{\prime}\left(h_{1}, \lambda\right) \chi_{1}\left(h_{1}, \lambda\right) \\
= & \left(\frac{\delta_{1}}{\gamma_{1}} \phi_{2}\left(h_{1}, \lambda\right)\right)\left(\frac{\delta_{2}}{\gamma_{2}} \chi_{2}^{\prime}\left(h_{1}, \lambda\right)\right) \\
& -\left(\frac{\delta_{2}}{\gamma_{2}} \phi_{2}^{\prime}\left(h_{1}, \lambda\right)\right)\left(\frac{\delta_{1}}{\gamma_{1}} \chi_{2}\left(h_{1}, \lambda\right)\right) \\
= & \frac{\delta_{1} \delta_{2}}{\gamma_{1} \gamma_{2}}(\lambda) \\
= & \left(\frac{\delta_{1} \delta_{3}}{\gamma_{1} \gamma_{3}} \phi_{3}\left(h_{2}, \lambda\right)\right)\left(\frac{\delta_{2} \delta_{4}}{\gamma_{2} \gamma_{4}} \chi_{3}^{\prime}\left(h_{2}, \lambda\right)\right) \\
& -\left(\frac{\delta_{2} \delta_{4}}{\gamma_{2} \gamma_{4}} \phi_{3}^{\prime}\left(h_{2}, \lambda\right)\right)\left(\frac{\delta_{1} \delta_{3}}{\gamma_{1} \gamma_{3}} \chi_{3}\left(h_{2}, \lambda\right)\right) \\
= & \frac{\delta_{1} \delta_{2} \delta_{3} \delta_{4}}{\gamma_{1} \gamma_{2} \gamma_{3} \gamma_{4}} \Delta_{3}(\lambda) .
\end{aligned}
$$


Corollary 4. The zeros of $\Delta_{1}(\lambda), \Delta_{2}(\lambda)$, and $\Delta_{3}(\lambda)$ coincide.

In view of Lemma 3 , we denote $\Delta_{1}(\lambda),\left(\delta_{1} \delta_{2} / \gamma_{1} \gamma_{2}\right) \Delta_{2}(\lambda)$, and $\left(\delta_{1} \delta_{2} \delta_{3} \delta_{4} / \gamma_{1} \gamma_{2} \gamma_{3} \gamma_{4}\right) \Delta_{3}(\lambda)$ by $\Delta(\lambda)$. Recalling the definitions of $\phi_{i}(x, \lambda)$ and $\chi_{i}(x, \lambda)$, we infer the next corollary.

Corollary 5. The function $\Delta(\lambda)$ is an entire function.

Theorem 6. The eigenvalues of (1)-(7) coincide with the zeros of $\Delta(\lambda)$.

Proof. Let $\Delta\left(\lambda_{0}\right)=0$. Then, $W\left(\phi_{1}\left(x, \lambda_{0}\right), \chi_{1}\left(x, \lambda_{0}\right)\right)=0$ for all $x \in\left[-1, h_{1}\right]$. Consequently, the functions $\phi_{1}\left(x, \lambda_{0}\right)$ and $\chi_{1}\left(x, \lambda_{0}\right)$ are linearly dependent; that is, $\chi_{1}\left(x, \lambda_{0}\right)=$ $k \phi_{1}\left(x, \lambda_{0}\right), x \in\left[-1, h_{1}\right]$, for some $k \neq 0$. By (21) and (22), from this equality, we have

$$
\chi^{\prime}\left(-1, \lambda_{0}\right)=k \phi_{1}^{\prime}\left(-1, \lambda_{0}\right)=0,
$$

and so $\chi\left(x, \lambda_{0}\right)$ satisfies the first boundary condition (2). Recalling that the solution $\chi\left(x, \lambda_{0}\right)$ also satisfies the other boundary condition ( 3 ) and transmission conditions (4)-(7), we conclude that $\chi\left(x, \lambda_{0}\right)$ is an eigenfunction of (1)-(7); that is, $\lambda_{0}$ is an eigenvalue. Thus, each zero of $\Delta(\lambda)$ is an eigenvalue. Now, let $\lambda_{0}$ be an eigenvalue, and let $u_{0}(x)$ be an eigenfunction with this eigenvalue. Suppose that $\Delta\left(\lambda_{0}\right) \neq 0$, whence $W\left(\phi_{1}\left(x, \lambda_{0}\right), \chi_{1}\left(x, \lambda_{0}\right)\right) \neq 0, W\left(\phi_{2}\left(x, \lambda_{0}\right), \chi_{2}\left(x, \lambda_{0}\right)\right) \neq 0$, and $W\left(\phi_{3}\left(x, \lambda_{0}\right), \chi_{3}\left(x, \lambda_{0}\right)\right) \neq 0$. From this, by virtue of the wellknown properties of Wronskians, it follows that each of the pairs $\phi_{1}\left(x, \lambda_{0}\right), \chi_{1}\left(x, \lambda_{0}\right) ; \phi_{2}\left(x, \lambda_{0}\right), \chi_{2}\left(x, \lambda_{0}\right)$ and $\phi_{3}\left(x, \lambda_{0}\right)$, $\chi_{3}\left(x, \lambda_{0}\right)$ is linearly independent. Therefore, the solution $u_{0}(x)$ of (1) may be represented as

$$
u_{0}(x)= \begin{cases}c_{1} \phi_{1}\left(x, \lambda_{0}\right)+c_{2} \chi_{1}\left(x, \lambda_{0}\right), & x \in\left[-1, h_{1}\right), \\ c_{3} \phi_{2}\left(x, \lambda_{0}\right)+c_{4} \chi_{2}\left(x, \lambda_{0}\right), & x \in\left(h_{1}, h_{2}\right), \\ c_{5} \phi_{3}\left(x, \lambda_{0}\right)+c_{6} \chi_{3}\left(x, \lambda_{0}\right), & x \in\left(h_{2}, 1\right],\end{cases}
$$

where at least one of the coefficients $c_{i}(i=\overline{1,6})$ is not zero. Considering the true equalities

$$
L_{v}\left(u_{0}(x)\right)=0, \quad v=\overline{1,6}
$$

as the homogenous system of linear equations in the variables $c_{i}(i=\overline{1,6})$ and taking (24), (25), (27), (28), (33), (34), (36), and (37) into account, we see that the determinant of this system is equal to $-\left(\left(\delta_{1} \delta_{2} \delta_{3} \delta_{4}\right)^{2} / \gamma_{1} \gamma_{2} \gamma_{3} \gamma_{4}\right) \Delta^{4}\left(\lambda_{0}\right)$, and so it does not vanish by assumption. Consequently, the system (41) has the only trivial solution $c_{i}=0(i=\overline{1,6})$. We thus get at a contradiction, which completes the proof.

Theorem 7. Let $\lambda=\mu^{2}$ and $\operatorname{Im} \mu=t$. Then, the following asymptotic equalities hold as $|\lambda| \rightarrow \infty$ :

$$
\begin{aligned}
\phi_{1}^{(k)}(x, \lambda)= & \frac{d^{k}}{d x^{k}} \cos \left[\mu \omega_{1}(x+1)\right] \\
& +O\left(\frac{1}{|\mu|^{1-k}} \exp \left(|t| \omega_{1}(x+1)\right)\right),
\end{aligned}
$$

$$
\begin{aligned}
\phi_{2}^{(k)}(x, \lambda)= & \frac{\gamma_{1}}{\delta_{1}} \frac{d^{k}}{d x^{k}} \cos \left[\mu\left(\omega_{2} x+\omega_{1} h_{1}+\omega_{1}\right)\right] \\
& +O\left(\frac{1}{|\mu|^{1-k}} \exp \left(|t|\left(\omega_{2} x+\omega_{1} h_{1}+\omega_{1}\right)\right)\right),
\end{aligned}
$$

$$
\begin{aligned}
\phi_{3}^{(k)}(x, \lambda)= & \frac{\gamma_{1} \gamma_{3}}{\delta_{1} \delta_{3}} \frac{d^{k}}{d x^{k}} \cos \left[\mu\left(\omega_{3} x+\omega_{2} h_{2}+\omega_{1}\right)\right] \\
& +O\left(\frac{1}{|\mu|^{1-k}} \exp \left(|t|\left(\omega_{3} x+\omega_{2} h_{2}+\omega_{1}\right)\right)\right),
\end{aligned}
$$

for $k=0$ and $k=1$. Moreover, each of these asymptotic equalities holds uniformly for $x$.

Proof. Asymptotic formulas for $\phi_{1}(x, \lambda)$ and $\phi_{2}(x, \lambda)$ are found in [9, Lemma 1.7] and [8, Theorem 3.2], respectively. But the formulas for $\phi_{3}(x, \lambda)$ need individual considerations, since this solution is defined by the initial condition with some special nonstandard form. The initial-value problem (26)-(28) can be transformed into the equivalent integral equation

$$
\begin{aligned}
u(x)= & \frac{\gamma_{3}}{\delta_{3}} \phi_{2}\left(h_{2}, \lambda\right) \cos \mu \omega_{3} x+\frac{\gamma_{4}}{\mu \omega_{3} \delta_{4}} \phi_{2}^{\prime}\left(h_{2}, \lambda\right) \sin \mu \omega_{3} x \\
& +\frac{\omega_{3}}{\mu} \int_{h_{2}}^{x} \sin \left[\mu \omega_{3}(x-y)\right] q(y) u(y) d y .
\end{aligned}
$$

Inserting (43) in (45), we have

$$
\begin{aligned}
\phi_{3}(x, \lambda)= & \frac{\gamma_{1} \gamma_{3}}{\delta_{1} \delta_{3}} \cos \left[\mu\left(\omega_{3} x+\omega_{2} h_{2}+\omega_{1}\right)\right] \\
& +\frac{\omega_{3}}{\mu} \int_{h_{2}}^{x} \sin \left[\mu \omega_{3}(x-y)\right] q(y) \phi_{3}(y, \lambda) d y \\
& +O\left(\frac{1}{|\mu|} \exp \left(|t|\left(\omega_{3} x+\omega_{2} h_{2}+\omega_{1}\right)\right)\right) .
\end{aligned}
$$

Multiplying this by $\exp \left(-|t|\left(\omega_{3} x+\omega_{2} h_{2}+\omega_{1}\right)\right)$ and denoting $F(x, \lambda)=\exp \left(-|t|\left(\omega_{3} x+\omega_{2} h_{2}+\omega_{1}\right)\right) \phi_{3}(x, \lambda)$, we have the next "asymptotic integral equation":

$$
\begin{aligned}
F(x, \lambda)= & \frac{\gamma_{1} \gamma_{3}}{\delta_{1} \delta_{3}} \exp \left(-|t|\left(\omega_{3} x+\omega_{2} h_{2}+\omega_{1}\right)\right) \\
& \times \cos \left[\mu\left(\omega_{3} x+\omega_{2} h_{2}+\omega_{1}\right)\right] \\
& +\frac{\omega_{3}}{\mu} \int_{h_{2}}^{x} \sin \left[\mu \omega_{3}(x-y)\right] \\
& \times \exp \left(-|t| \omega_{3}(x-y)\right) q(y) F(y, \lambda) d y \\
& +O\left(\frac{1}{\mu}\right) .
\end{aligned}
$$

Putting $M(\lambda)=\max _{x \in\left[h_{2}, 1\right]}|F(x, \lambda)|$, from the last equation, we derive that

$$
M(\lambda) \leq M_{0}\left(\left|\frac{\gamma_{1} \gamma_{3}}{\delta_{1} \delta_{3}}\right|+\frac{1}{\mu}\right),
$$


for some $M_{0}>0$. Consequently, $M(\lambda)=O(1)$ as $|\lambda| \rightarrow \infty$, and so $\phi_{3}(x, \lambda)=O\left(\exp \left(|t|\left(\omega_{3} x+\omega_{2} h_{2}+\omega_{1}\right)\right)\right)$ as $|\lambda| \rightarrow \infty$. Inserting the integral term of (46) yields (44) for $k=0$. The case $k=1$ of (44) follows at once on differentiating (43) and making the same procedure as in the case $k=0$.

Theorem 8. Let $\lambda=\mu^{2}, \mu=\sigma+i t$. Then, the following asymptotic formula holds for the eigenvalues of the boundary value transmission problem (1)-(7):

$$
\mu_{n}=\frac{\pi(n-1)}{\omega_{3}+\omega_{2} h_{2}+\omega_{1}}+O\left(\frac{1}{n}\right) .
$$

Proof. Putting $x=1$ in $\Delta_{3}(\lambda)=\phi_{3}(x, \lambda) \chi_{3}^{\prime}(x, \lambda)-$ $\phi_{3}^{\prime}(x, \lambda) \chi_{3}(x, \lambda)$ and inserting $\chi_{3}(1, \lambda)=\beta_{2}^{\prime} \lambda+\beta_{2}, \chi_{3}^{\prime}(1, \lambda)=$ $\beta_{1}^{\prime} \lambda+\beta_{1}$, we have the following representation for $\Delta_{3}(\lambda)$ :

$$
\Delta_{3}(\lambda)=\left(\beta_{1}^{\prime} \lambda+\beta_{1}\right) \phi_{3}(1, \lambda)-\left(\beta_{2}^{\prime} \lambda+\beta_{2}\right) \phi_{3}^{\prime}(1, \lambda) .
$$

Putting $x=1$ in (44) and inserting the result in (50), we derive now that

$$
\begin{aligned}
\Delta_{3}(\lambda)= & \frac{\delta_{2} \delta_{4}}{\gamma_{2} \gamma_{4}} \omega_{3} \beta_{2}^{\prime} \mu^{3} \sin \left[\mu\left(\omega_{3}+\omega_{2} h_{2}+\omega_{1}\right)\right] \\
& +O\left(|\mu|^{2} \exp \left(2|t|\left(\omega+\omega_{2} h_{2}+\omega_{1}\right)\right)\right) .
\end{aligned}
$$

By applying the well-known Rouché theorem which asserts that if $f(z)$ and $g(z)$ are analytic inside and on a closed contour $\Gamma$ and $|g(z)|<|f(z)|$ on $\Gamma$, then $f(z)$ and $f(z)+g(z)$ have the same number of zeros inside $\Gamma$ provided that the zeros are counted with multiplicity on a sufficiently large contour, it follows that $\Delta_{3}(\lambda)$ has the same number of zeros inside the contour as the leading term in (51). Hence, if $\lambda_{0}<$ $\lambda_{1}<\lambda_{2} \ldots$ are the zeros of $\Delta_{3}(\lambda)$ and $\mu_{n}^{2}=\lambda_{n}$, we have

$$
\frac{\pi(n-1)}{\omega_{3}+\omega_{2} h_{2}+\omega_{1}}+\delta_{n}
$$

for sufficiently large $n$, where $\left|\delta_{n}\right|<\pi / 4\left(\omega_{3}+\omega_{2} h_{2}+\omega_{1}\right)$ for sufficiently large $n$. By putting in (51), we have $\delta_{n}=O(1 / n)$, and the proof is completed.

Theorem 9. The following asymptotic formula holds for the eigenfunctions

$$
\phi_{\lambda_{n}}(x)= \begin{cases}\phi_{1}\left(x, \lambda_{n}\right), & x \in\left[-1, h_{1}\right), \\ \phi_{2}\left(x, \lambda_{n}\right), & x \in\left(h_{1}, h_{2}\right), \\ \phi_{3}\left(x, \lambda_{n}\right), & x \in\left(h_{2}, 1\right],\end{cases}
$$

of (1)-(7):

$$
\phi_{\lambda_{n}}(x)= \begin{cases}\cos \left[\frac{\omega_{1} \pi(n-1)(x+1)}{\omega_{2}+\omega_{1}}\right]+O\left(\frac{1}{n}\right), & x \in\left[-1, h_{1}\right), \\ \frac{\gamma_{1}}{\delta_{1}} \cos \left[\frac{\left(\omega_{2} x+\omega_{1} h_{1}+\omega_{1}\right) \pi(n-1)}{\omega_{2}+\omega_{1} h_{1}+\omega_{1}}\right]+O\left(\frac{1}{n}\right), & x \in\left(h_{1}, h_{2}\right), \\ \frac{\gamma_{1} \gamma_{3}}{\delta_{1} \delta_{3}} \cos \left[\frac{\left(\omega_{3} x+\omega_{2} h_{2}+\omega_{1}\right) \pi(n-1)}{\omega_{3}+\omega_{2} h_{2}+\omega_{1}}\right]+O\left(\frac{1}{n}\right), & x \in\left(h_{2}, 1\right] .\end{cases}
$$

Proof. Inserting (44) in the integral term of (51), we easily see that

$$
\begin{gathered}
\int_{h_{2}}^{x} \sin \left[\mu \omega_{3}(x-y)\right] q(y) \phi_{3}(y, \lambda) d y \\
\quad=O\left(\exp \left(|t|\left(\omega_{3} x+\omega_{2} h_{2}+\omega_{1}\right)\right)\right)
\end{gathered}
$$

Inserting in (42) yields

$$
\begin{aligned}
\phi_{3}(x, \lambda)= & \frac{\gamma_{1} \gamma_{3}}{\delta_{1} \delta_{3}} \cos \left[\mu\left(\omega_{3} x+\omega_{2} h_{2}+\omega_{1}\right)\right] \\
& +O\left(\frac{1}{|\mu|} \exp |t|\left(\omega_{3} x+\omega_{2} h_{2}+\omega_{1}\right)\right) .
\end{aligned}
$$

We already know that all eigenvalues are real. Furthermore, putting $\lambda=-H, H>0$ in (51), we infer that $\omega(-H) \rightarrow \infty$ as $H \rightarrow+\infty$, and so $\omega(-H) \neq 0$ for sufficiently large $R>$ 0 . Consequently, the set of eigenvalues is bounded below. Letting $\sqrt{\lambda_{n}}=\mu_{n}$ in (56), we now obtain

$$
\phi_{3}\left(x, \lambda_{n}\right)=\frac{\gamma_{1} \gamma_{3}}{\delta_{1} \delta_{3}} \cos \left[\mu_{n}\left(\omega_{3} x+\omega_{2} h_{2}+\omega_{1}\right)\right]+O\left(\frac{1}{\mu_{n}}\right)
$$

since $t_{n}=\operatorname{lm} \mu_{n}$ for sufficiently large $n$. After some calculation, we easily see that

$$
\begin{aligned}
\cos & {\left[\mu_{n}\left(\omega_{3} x+\omega_{2} h_{2}+\omega_{1}\right)\right] } \\
& =\cos \left[\frac{\left(\omega_{3} x+\omega_{2} h_{2}+\omega_{1}\right) \pi(n-1)}{\omega_{3}+\omega_{2} h_{2}+\omega_{1}}\right]+O\left(\frac{1}{n}\right) .
\end{aligned}
$$


Consequently,

$$
\begin{aligned}
\phi_{3}\left(x, \lambda_{n}\right)= & \frac{\gamma_{1} \gamma_{3}}{\delta_{1} \delta_{3}} \cos \left[\frac{\left(\omega_{3} x+\omega_{2} h_{2}+\omega_{1}\right) \pi(n-1)}{\omega_{3}+\omega_{2} h_{2}+\omega_{1}}\right] \\
& +O\left(\frac{1}{n}\right) .
\end{aligned}
$$

In a similar method, we can deduce that

$$
\begin{aligned}
& \begin{aligned}
& \phi_{2}\left(x, \lambda_{n}\right)= \frac{\gamma_{1}}{\delta_{1}} \cos \left[\frac{\left(\omega_{2} x+\omega_{1} h_{1}+\omega_{1}\right) \pi(n-1)}{\omega_{2}+\omega_{1} h_{1}+\omega_{1}}\right] \\
&+O\left(\frac{1}{n}\right), \\
& \phi_{1}\left(x, \lambda_{n}\right)=\cos \left[\frac{\omega_{1} \pi(n-1)(x+1)}{\omega_{2}+\omega_{1}}\right]+O\left(\frac{1}{n}\right) .
\end{aligned}
\end{aligned}
$$

Thus is the proof of the theorem, completed.

\section{Asymptotic Formulas for Normalized Eigenfunctions}

We will first obtain the norms of the eigenelements

$$
\Phi_{n}:=\left(\begin{array}{c}
\phi_{\lambda_{n}}(x) \\
R_{1}^{\prime}\left(\phi_{\lambda_{n}}\right)
\end{array}\right)
$$

It is evident that the two-component vectors

$$
\Phi_{n}:=\left(\begin{array}{c}
\phi_{\lambda_{n}}(x) \\
R_{1}^{\prime}\left(\phi_{\lambda_{n}}\right)
\end{array}\right), \quad n=0,1,2, \ldots
$$

are the eigenelements of $A$ with eigenvalue $\lambda_{n}$. For $n \neq m$,

$$
\left\langle\Phi_{n}, \Phi_{m}\right\rangle_{H}=0, \quad n, m=0,1,2, \ldots
$$

since $A$ is symmetric. Putting

$$
\psi_{n}:=\frac{\phi_{\lambda_{n}}(x)}{\left\|\Phi_{n}\right\|_{H}}
$$

we see easily that the eigenelements

$$
\Psi_{n}=\left(\begin{array}{c}
\psi_{\lambda_{n}}(x) \\
R_{1}^{\prime}\left(\psi_{\lambda_{n}}\right)
\end{array}\right), \quad n, m=0,1,2, \ldots
$$

are orthonormal. That is, $A \Psi_{n}=\lambda_{n} \Psi_{n}$ and $\left\langle\Psi_{n}, \Psi_{m}\right\rangle_{H}=\delta_{n m}$, where $\delta_{n m}$ is the Kronecker delta.
Lemma 10. The following asymptotic equality holds:

$$
R_{1}^{\prime}\left(\phi_{\lambda_{n}}\right)=O\left(\frac{1}{n}\right)
$$

Proof. It follows that from the equalities $\Delta_{3}\left(\lambda_{n}\right)=0$ that

$$
\lambda_{n} R_{1}^{\prime}\left(\phi_{3 \lambda_{n}}\right)+R_{1}\left(\phi_{3 \lambda_{n}}\right)=0
$$

From (44), we obtain

$$
\begin{aligned}
R_{1}\left(\phi_{3 \lambda_{n}}\right) & =\beta_{1} \phi_{3 \lambda_{n}}(1)-\beta_{2} \phi_{3 \lambda_{n}}^{\prime}(1) \\
& =\beta_{1} O(1)-\beta_{2} O\left(\left|s_{n}\right|\right)
\end{aligned}
$$

Applying Theorem 8, we now have

$$
R_{1}\left(\phi_{3 \lambda_{n}}\right)=O(n)
$$

Inserting (69) in (67) and using Theorem 8, we find

$$
R_{1}^{\prime}\left(\phi_{3 \lambda_{n}}\right)=-\frac{1}{\lambda_{n}} R_{1}\left(\phi_{3 \lambda_{n}}\right)=O\left(\frac{1}{n}\right)
$$

The proof is completed.

Theorem 11. Let $\Phi_{n}$ be defined as in (61). Then, the following asymptotic formula holds for the norms $\left\|\Phi_{n}\right\|_{H}$ of the eigenelements $\Phi_{n}$ :

$$
\left\|\Phi_{n}\right\|_{H}=\sqrt{\frac{\omega_{1}^{2} \delta_{1} \delta_{3} \gamma_{2} \gamma_{4}+\omega_{2}^{2} \delta_{2} \delta_{3} \gamma_{1} \gamma_{4}+\omega_{3}^{2} \delta_{2} \delta_{4} \gamma_{1} \gamma_{3}}{2 \delta_{1} \delta_{3} \gamma_{2} \gamma_{4}}}
$$

$$
+O\left(\frac{1}{n}\right)
$$

Proof. By (54), we have

$$
\begin{gathered}
\int_{-1}^{h_{1}}\left(\phi_{\lambda_{n}}(x)\right)^{2} d x=\frac{1}{2}+O\left(\frac{1}{n}\right), \\
\int_{h_{1}}^{h_{2}}\left(\phi_{\lambda_{n}}(x)\right)^{2} d x=\frac{\gamma_{1}^{2}}{2 \delta_{1}^{2}}+O\left(\frac{1}{n}\right), \\
\int_{h_{2}}^{1}\left(\phi_{\lambda_{n}}(x)\right)^{2} d x=\frac{\gamma_{1}^{2}}{2 \delta_{1}^{2}} \frac{\gamma_{3}^{2}}{\delta_{3}^{2}}+O\left(\frac{1}{n}\right) .
\end{gathered}
$$


Using (66), (72), (73), and (74), we obtain

$$
\begin{aligned}
\left\|\Phi_{n}\right\|_{H}^{2}= & \omega_{1}^{2} \int_{-1}^{h_{1}}\left(\phi_{\lambda_{n}}(x)\right)^{2} d x+\omega_{2}^{2} \frac{\delta_{1} \delta_{2}}{\gamma_{1} \gamma_{2}} \int_{h_{1}}^{h_{2}}\left(\phi_{\lambda_{n}}(x)\right)^{2} d x \\
& +\omega_{3}^{2} \frac{\delta_{1} \delta_{2} \delta_{3} \delta_{4}}{\gamma_{1} \gamma_{2} \gamma_{3} \gamma_{4}} \int_{h_{2}}^{1}\left(\phi_{\lambda_{n}}(x)\right)^{2} d x \\
& +\frac{\delta_{1} \delta_{2} \delta_{3} \delta_{4}}{\rho \gamma_{1} \gamma_{2} \gamma_{3} \gamma_{4}}\left(R_{1}^{\prime}\left(\phi_{\lambda_{n}}\right)\right)^{2} \\
= & \omega_{1}^{2}\left[\frac{1}{2}+O\left(\frac{1}{n}\right)\right]+\omega_{2}^{2} \frac{\delta_{1} \delta_{2}}{\gamma_{1} \gamma_{2}}\left[\frac{\gamma_{1}^{2}}{2 \delta_{1}^{2}}+O\left(\frac{1}{n}\right)\right] \\
& +\omega_{3}^{2} \frac{\delta_{1} \delta_{2} \delta_{3} \delta_{4}}{\gamma_{1} \gamma_{2} \gamma_{3} \gamma_{4}}\left[\frac{\gamma_{1}^{2}}{2 \delta_{1}^{2}} \frac{\gamma_{3}^{2}}{\delta_{3}^{2}}+O\left(\frac{1}{n}\right)\right] \\
& +\frac{\delta_{1} \delta_{2} \delta_{3} \delta_{4}}{\rho \gamma_{1} \gamma_{2} \gamma_{3} \gamma_{4}} O\left(\frac{1}{n^{2}}\right)
\end{aligned}
$$$$
=\frac{\left(\omega_{1}^{2} \delta_{1} \delta_{3} \gamma_{2} \gamma_{4}+\omega_{2}^{2} \delta_{2} \delta_{3} \gamma_{1} \gamma_{4}+\omega_{3}^{2} \delta_{2} \delta_{4} \gamma_{1} \gamma_{3}\right)}{2 \delta_{1} \delta_{3} \gamma_{2} \gamma_{4}}
$$$$
+O\left(\frac{1}{n}\right)
$$

Consequently,

$$
\begin{aligned}
\left\|\Phi_{n}\right\|_{H}= & \sqrt{\frac{\omega_{1}^{2} \delta_{1} \delta_{3} \gamma_{2} \gamma_{4}+\omega_{2}^{2} \delta_{2} \delta_{3} \gamma_{1} \gamma_{4}+\omega_{3}^{2} \delta_{2} \delta_{4} \gamma_{1} \gamma_{3}}{2 \delta_{1} \delta_{3} \gamma_{2} \gamma_{4}}} \\
& +O\left(\frac{1}{n}\right),
\end{aligned}
$$

which proves (71).

Theorem 12. The first components of the normalized eigenelements (65) have the following asymptotic representation as $n \rightarrow \infty:$

$$
\psi_{n}(x)= \begin{cases}\sqrt{\frac{2 \delta_{1} \delta_{3} \gamma_{2} \gamma_{4}}{\omega_{1}^{2} \delta_{1} \delta_{3} \gamma_{2} \gamma_{4}+\omega_{2}^{2} \delta_{2} \delta_{3} \gamma_{1} \gamma_{4}+\omega_{3}^{2} \delta_{2} \delta_{4} \gamma_{1} \gamma_{3}}} \cos \left(\frac{\omega_{1} \pi(n-1)(x+1)}{\omega_{1}+\omega_{2}}\right)+O\left(\frac{1}{n}\right), & x \in\left[-1, h_{1}\right), \\ \frac{\gamma_{1}}{\delta_{1}} \sqrt{\frac{2 \delta_{1} \delta_{3} \gamma_{2} \gamma_{4}}{\omega_{1}^{2} \delta_{1} \delta_{3} \gamma_{2} \gamma_{4}+\omega_{2}^{2} \delta_{2} \delta_{3} \gamma_{1} \gamma_{4}+\omega_{3}^{2} \delta_{2} \delta_{4} \gamma_{1} \gamma_{3}}} \cos \left(\frac{\pi(n-1)\left(\omega_{2} x+\omega_{1} h_{1}+\omega_{1}\right)}{\omega_{2}+\omega_{1} h_{1}+\omega_{1}}\right)+O\left(\frac{1}{n}\right), & x \in\left(h_{1}, h_{2}\right), \\ \frac{\gamma_{1}}{\delta_{1}} \frac{\gamma_{3}}{\delta_{3}} \sqrt{\frac{2 \delta_{1} \delta_{3} \gamma_{2} \gamma_{4}}{\omega_{1}^{2} \delta_{1} \delta_{3} \gamma_{2} \gamma_{4}+\omega_{2}^{2} \delta_{2} \delta_{3} \gamma_{1} \gamma_{4}+\omega_{3}^{2} \delta_{2} \delta_{4} \gamma_{1} \gamma_{3}}} \cos \left(\frac{\pi(n-1)\left(\omega_{3} x+\omega_{2} h_{2}+\omega_{1}\right)}{\omega_{3}+\omega_{2} h_{2}+\omega_{1}}\right)+O\left(\frac{1}{n}\right), & x \in\left(h_{2}, 1\right] .\end{cases}
$$

Proof. From (71), it follows that

$$
\begin{aligned}
\frac{1}{\left\|\Phi_{n}\right\|_{H}}= & \sqrt{\frac{2 \delta_{1} \delta_{3} \gamma_{2} \gamma_{4}}{\omega_{1}^{2} \delta_{1} \delta_{3} \gamma_{2} \gamma_{4}+\omega_{2}^{2} \delta_{2} \delta_{3} \gamma_{1} \gamma_{4}+\omega_{3}^{2} \delta_{2} \delta_{4} \gamma_{1} \gamma_{3}}} \\
& +O\left(\frac{1}{n}\right) .
\end{aligned}
$$

Putting (54) and (78) in (64), we find the needed asymptotic formula (77).

\section{Green's Function}

Let $A: H \rightarrow H$ be defined by (10) and (11), and let $\lambda$ not be an eigenvalue of $A$. For finding the resolvent operator $R(\lambda, A)=$ $(\lambda I-A)^{-1}$, consider the operator equation

$$
(\lambda I-A) U=F
$$

for $F=\left(\begin{array}{c}f(x) \\ f_{1}\end{array}\right) \in H$. This operator equation is equivalent to the inhomogeneous differential equation

$$
\begin{array}{r}
u^{\prime \prime}+(\lambda \omega(x)-q(x)) u=f(x) \\
\text { for } x \in\left[-1, h_{1}\right) \cup\left(h_{1}, h_{2}\right) \cup\left(h_{2}, 1\right]
\end{array}
$$

subject to the inhomogeneous boundary conditions

$$
\begin{gathered}
u^{\prime}(-1)=0, \\
\lambda\left(\beta_{1}^{\prime} u(1)-\beta_{2}^{\prime} u^{\prime}(1)\right)+\left(\beta_{1} u(1)-\beta_{2} u^{\prime}(1)\right)=f_{1}
\end{gathered}
$$

and the homogeneous transmission conditions

$$
\begin{gathered}
\gamma_{1} u\left(h_{1}-0\right)-\delta_{1} u\left(h_{1}+0\right)=0, \\
\gamma_{2} u^{\prime}\left(h_{1}-0\right)-\delta_{2} u^{\prime}\left(h_{1}+0\right)=0, \\
\gamma_{3} u\left(h_{2}-0\right)-\delta_{3} u\left(h_{2}+0\right)=0, \\
\gamma_{4} u^{\prime}\left(h_{2}-0\right)-\delta_{4} u^{\prime}\left(h_{2}+0\right)=0 .
\end{gathered}
$$

By applying the same techniques as in [8], we can prove that the problem $(80)-(82)$ has a unique solution $u(x, \lambda)$ which can be represented as 


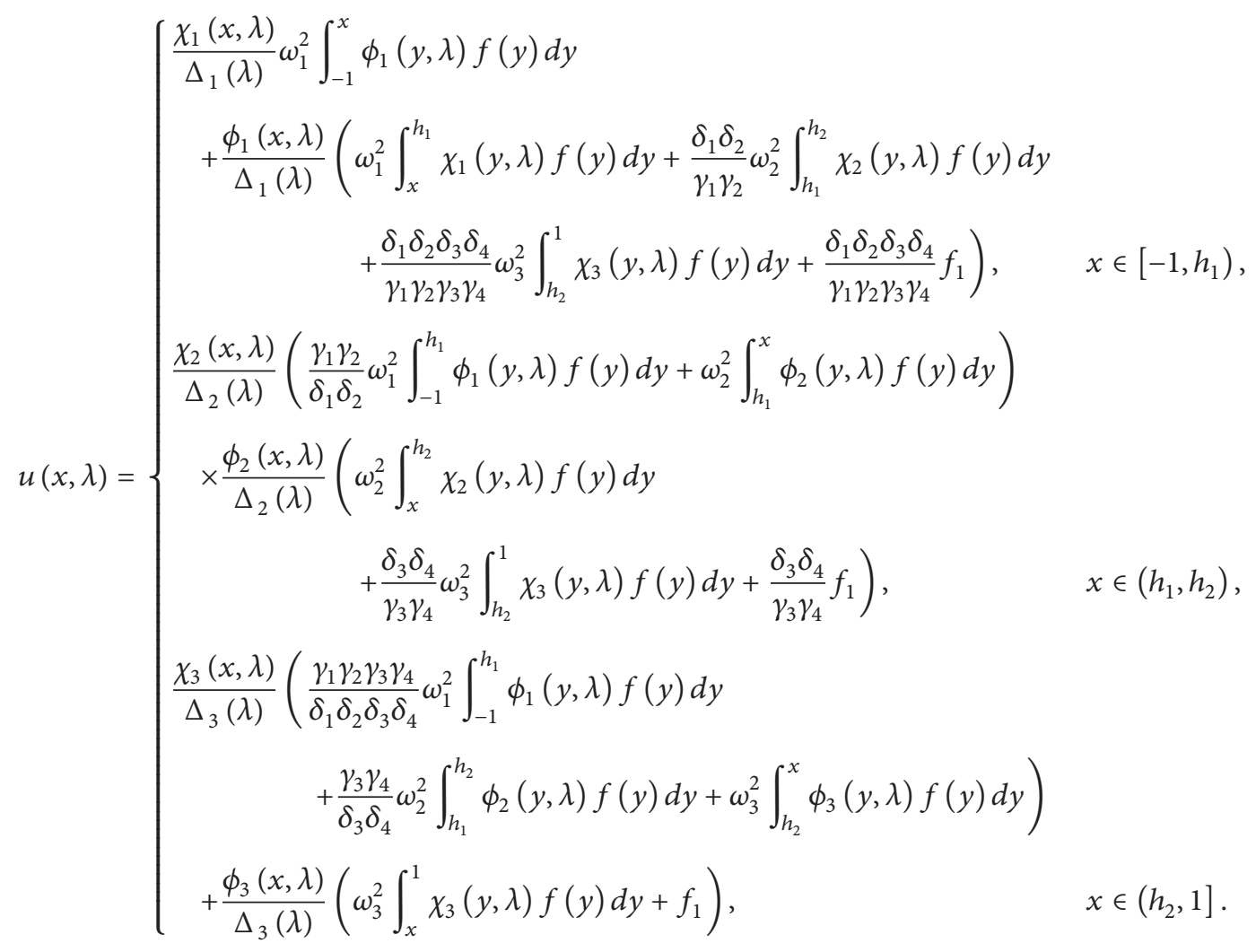

Putting

$$
G(x, y, \lambda)= \begin{cases}\frac{\chi(x, \lambda) \phi(y, \lambda)}{\omega(\lambda)} & \text { for }-1 \leq y \leq x \leq 1, \\ \frac{\phi(x, \lambda) \chi(y, \lambda)}{\omega(\lambda)} & \text { for }-1 \leq x \leq y \leq 1,\end{cases}
$$

where $x \neq 0$ and $y \neq 0$, we reduce (73) to

$$
\begin{aligned}
u(x, \lambda)= & \omega_{1}^{2} \int_{-1}^{h_{1}} G(x, y, \lambda) f(y) d y \\
& +\omega_{2}^{2} \frac{\delta_{1} \delta_{2}}{\gamma_{1} \gamma_{2}} \int_{h_{1}}^{h_{2}} G(x, y, \lambda) f(y) d y \\
& +\omega_{3}^{2} \frac{\delta_{1} \delta_{2} \delta_{3} \delta_{4}}{\gamma_{1} \gamma_{2} \gamma_{3} \gamma_{4}} \int_{h_{2}}^{1} G(x, y, \lambda) f(y) d y+f_{1} \frac{\phi(x, \lambda)}{\omega(\lambda)} .
\end{aligned}
$$

On the other hand, by applying $R_{1}^{\prime}$ to Green's function with respect to the variable $y$ and recalling that $\chi(x, \lambda)$ satisfies the initial conditions (30) and (31), we have

$$
\begin{aligned}
R_{1}^{\prime}(G(x, \cdot, \lambda)) & =\beta_{1}^{\prime} G(x, 1, \lambda)-\beta_{2}^{\prime} \frac{\partial G(x, 1, \lambda)}{\partial y} \\
& =\frac{\gamma_{1} \gamma_{2} \gamma_{3} \gamma_{4}}{\delta_{1} \delta_{2} \delta_{3} \delta_{4}} \frac{\phi(x, \lambda)}{\omega(\lambda)}\left(\beta_{1}^{\prime} \chi(1, \lambda)-\beta_{2}^{\prime} \chi^{\prime}(1, \lambda)\right)
\end{aligned}
$$

$$
\begin{aligned}
= & \frac{\gamma_{1} \gamma_{2} \gamma_{3} \gamma_{4}}{\delta_{1} \delta_{2} \delta_{3} \delta_{4}} \frac{\phi(x, \lambda)}{\omega(\lambda)} \\
& \times\left(\beta_{1}^{\prime}\left(\beta_{2}^{\prime} \lambda+\beta_{2}\right)-\beta_{2}^{\prime}\left(\beta_{1}^{\prime} \lambda+\beta_{1}\right)\right) \\
= & \frac{\rho \gamma_{1} \gamma_{2} \gamma_{3} \gamma_{4}}{\delta_{1} \delta_{2} \delta_{3} \delta_{4}} \frac{\phi(x, \lambda)}{\omega(\lambda)}
\end{aligned}
$$

Inserting in (85) yields

$$
\begin{aligned}
u(x, \lambda)= & \omega_{1}^{2} \int_{-1}^{h_{1}} G(x, y, \lambda) f(y) d y \\
& +\omega_{2}^{2} \frac{\delta_{1} \delta_{2}}{\gamma_{1} \gamma_{2}} \int_{h_{1}}^{h_{2}} G(x, y, \lambda) f(y) d y \\
& +\omega_{3}^{2} \frac{\delta_{1} \delta_{2} \delta_{3} \delta_{4}}{\gamma_{1} \gamma_{2} \gamma_{3} \gamma_{4}} \int_{h_{2}}^{1} G(x, y, \lambda) f(y) d y \\
& +\frac{f_{1} \delta_{1} \delta_{2} \delta_{3} \delta_{4}}{\rho \gamma_{1} \gamma_{2} \gamma_{3} \gamma_{4}} R_{1}^{\prime}(G(x, \cdot, \lambda)) .
\end{aligned}
$$

We now introduce

$$
G_{x, \lambda}=\left(\begin{array}{c}
G(x, \cdot \lambda) \\
R_{1}^{\prime}(G(x, \cdot, \lambda))
\end{array}\right),
$$


which we will call Green's element of (80)-(82). The last formula (87) then takes the form

$$
u(x, \lambda)=\left\langle G_{x, \lambda}, \bar{F}\right\rangle
$$

where by $\bar{F}$ we mean $\bar{F}=\left(\begin{array}{c}\overline{f(x)} \\ \overline{f_{1}}\end{array}\right)$.

\section{References}

[1] E. C. Titchmarsh, Eigenfunctions Expansion Associated with Second Order Differential Equations, part 1, Oxford University Press, London, UK, 1962.

[2] J. Walter, "Regular eigenvalue problems with eigenvalue parameter in the boundary condition," Mathematische Zeitschrift, vol. 133, no. 4, pp. 301-312, 1973.

[3] D. B. Hinton, "An expansion theorem for an eigenvalue problem with eigenvalue parameter in the boundary condition," Quarterly Journal of Mathematics, vol. 30, no. 1, pp. 33-42, 1979.

[4] M. Kadakal and O. Sh. Mukhtarov, "Eigenvalues and eigenfunctions of Sturm-Liouville problem with two-point discontinuities containing eigenparameter-dependent boundary conditions," Scientia Magna, vol. 6, no. 4, pp. 33-48, 2010.

[5] M. Kadakal and O. Sh. Mukhtarov, "Discontinuous SturmLiouville problems containing eigenparameter in the boundary conditions," Acta Mathematica Sinica, vol. 22, no. 5, pp. 15191528, 2006.

[6] O. Sh. Mukhtarov and M. Kadakal, "On a Sturm-Liouville type problem with discontinuous in two-points," Far East Journal of Applied Mathematics, vol. 19, no. 3, pp. 337-352, 2005.

[7] O. Sh. Mukhtarov, M. Kandemir, and N. Kuruoğlu, "Distribution of eigenvalues for the discontinuous boundary-value problem with functional-manypoint conditions," Israel Journal of Mathematics, vol. 129, pp. 143-156, 2002.

[8] O. Sh. Mukhtarov and M. Kadakal, "Some spectral properties of one Sturm-Liouville type problem with discontinuous weight," Siberian Mathematical Journal, vol. 46, no. 4, pp. 681-694, 2005.

[9] C. T. Fulton, "Two-point boundary value problems with eigenvalue parameter contained in the boundary conditions," Proceedings of the Royal Society of Edinburgh A, vol. 77, no. 3-4, pp. 293-308, 1977.

[10] E. Bairamov and E. Ugurlu, "On the characteristic values of the real component of a dissipative boundary value transmission problem," Applied Mathematics and Computation, vol. 218, no. 19, pp. 9657-9663, 2012.

[11] A. N. Tikhonov and A. A. Samarskii, Equations of Mathematical Physics, Pergamon, New York, NY, USA, 1963.

[12] E. Şen, J. Seo, and S. Araci, "Asymptotic behaviour of eigenvalues and eigenfunctions of a Sturm-Liouville problem with retarded argument," Journal of Applied Mathematics, vol. 2013, Article ID 306917, 8 pages, 2013.

[13] E. Şen and A. Bayramov, "Calculation of eigenvalues and eigenfunctions of a discontinuous boundary value problem with retarded argument which contains a spectral parameter in the boundary condition," Mathematical and Computer Modelling, vol. 54, no. 11-12, pp. 3090-3097, 2011.

[14] E. Şen and A. Bayramov, "Asymptotic formulations of the eigenvalues and eigenfunctions for a boundary value problem," Mathematical Methods in the Applied Sciences, vol. 36, no. 12, pp. 1512-1519, 2013.
[15] Q. Yang and W. Wang, "Asymptotic behavior of a differential operator with discontinuities at two points," Mathematical Methods in the Applied Sciences, vol. 34, no. 4, pp. 373-383, 2011.

[16] Q. Yang and W. Wang, "Spectral properties of Sturm-Liouville operators with discontinuities at finite points," Mathematical Sciences, vol. 6, no. 34, 9 pages, 2012. 

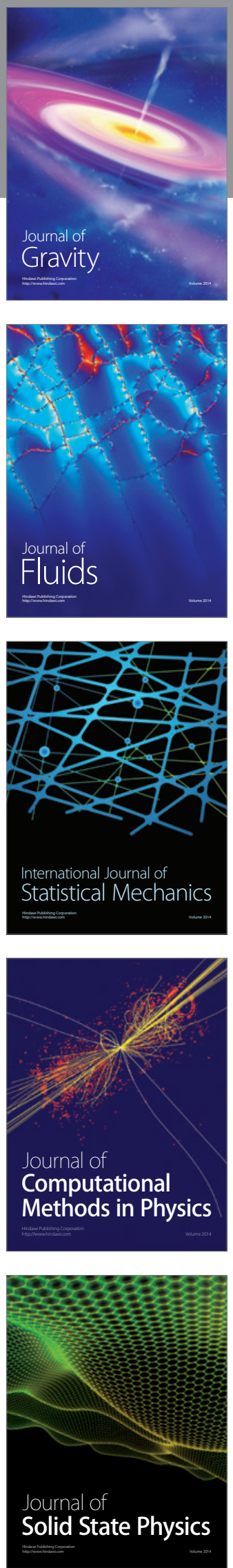

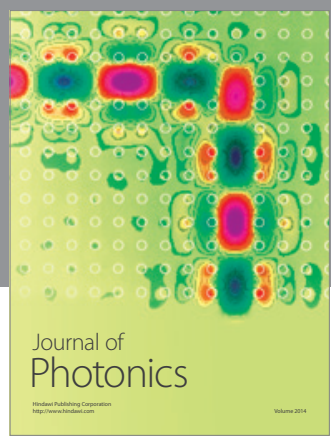

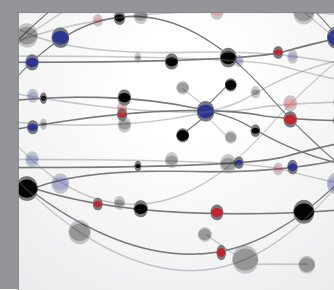

The Scientific World Journal

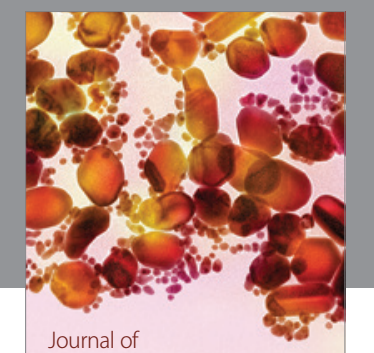

Soft Matter
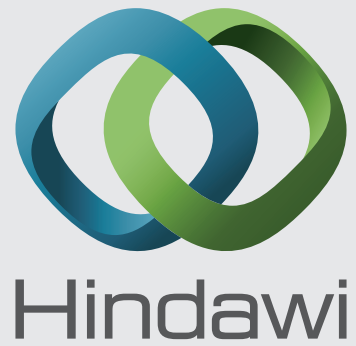

Submit your manuscripts at

http://www.hindawi.com
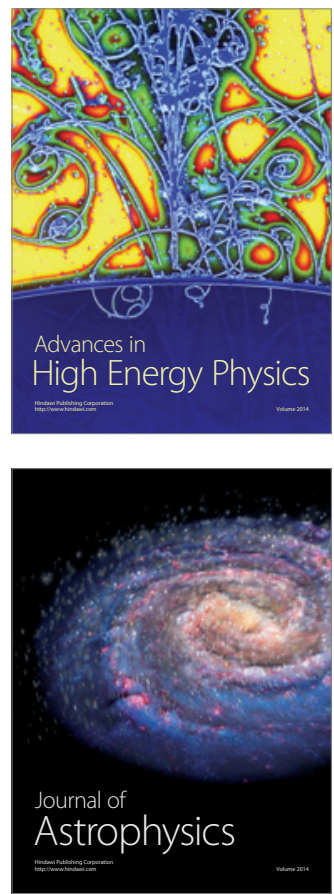
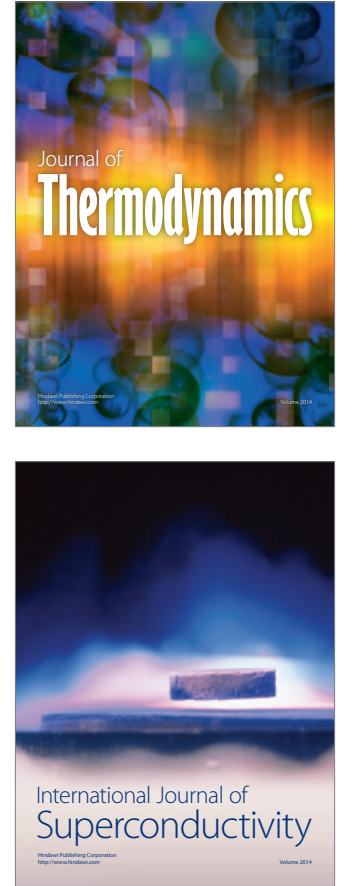
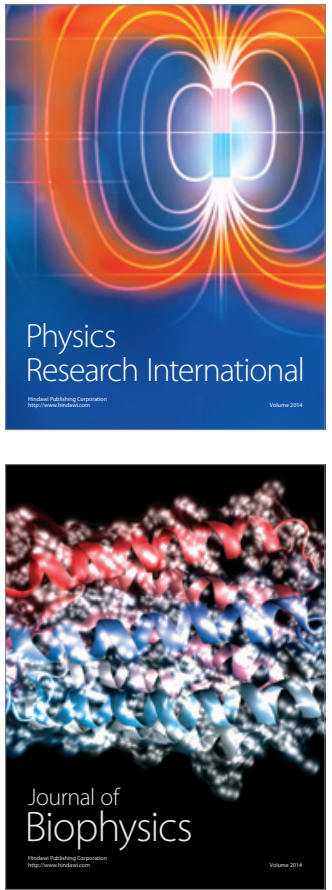
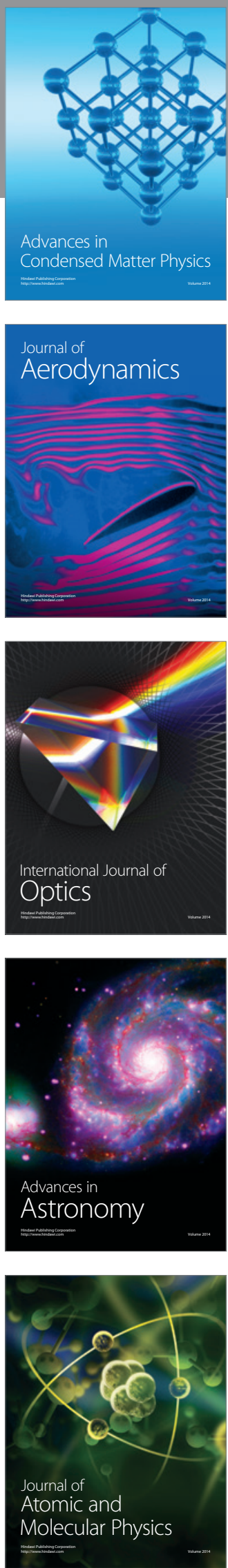